\title{
Adaptive High Stability modeling for Analysis of Micro grid for DC Converters
}

\author{
\#Rajender Boini \\ \#Research scholar, Department of Electrical Engineering, \\ Sunrise university, Alwar,Rajasthan.
}

\begin{abstract}
This paper presents the analysis of dc network in a hybrid micro grid system. A hybrid micro grid consist if ac and dc sub grids inter connected through multi port power electronic interface (MPEIs). For modeling a multiple switching converter an averaging method is implemented. Pole zero analysis of a small signal model is performed for analyzing the stability of the network. Simulation results are verified. Index Keywords:- Multiport power electronic interface, averaging method, pole zero analysis, small signal model.
\end{abstract}

\section{INTRODUCTION}

Due to the sustainable source of energy, the electricity market has been encountered a growth in distributed generations (DGs). There may be some technical challenges while integrating renewable sources with distribution grids. The available structure is not intended for distributed and deregulated forms. Hence some new methodologies and hardware are implemented to perform this transformation. A micro grid can combine distributed generation and energy storage equipments by superior control system. It can run with or without distributed power system. The converter acts as a bridge of micro power supply and the role of is effective for stable operation. Here decentralized control techniques are used for a micro grid operation. Owing to growing demand for good efficiency and speedy communication system, power switching centers with clean and sustainable forms of energy is of notice. This can be achieved by introducing DC distribution networks for DC applications with high performance. By eliminating ac to dc front end converter dc distribution network can promote the data centers. By removing these converters the efficiency can be improved and also increases the reliability of the system. The front end ac to dc converter can be replaced with multi port power electronic interface (MPEI) to benefit from distribution generation. Typically, individual ac to dc converters are used in an ac micro grid for storage systems, dc loads and distribution generations which are need to be connected with ac bus. The demand in ac and dc distribution network can be addressed simultaneously through hybrid ac de micro grid. Hybrid micro grid reduces the number of ac dc converters used in individual grids. But in many systems, in between ac and dc distribution networks only one point of common coupling (PCC) is used. Mostly solid state transformers (SST) are used as PCC. In this paper, multi port power electronic interface is introduced into the hybrid micro grid. Multiple point of coupling between ac and dc network is used to increase the controllability, reliability and performance of the hybrid microgrid. As power electronic applications is not effective for modeling a system with various switching frequencies, an extended averaging method is proposed in this paper which can model a microgrid dominated with switching converters. Pole zero stability of the system is also studied using this model. Finally stability analysis performance is analyzed using simulation result. 2. HYBRID MICRO GRID BASED ON MPEI In recent years new technologies have been emerged to have a more flexible and reliable power grid. The cost of migration from the existing distribution network structure with be more effective power grid, a smart grid can be introduced. Thus the cost of infrastructure replacement can be reduced. While, when the micro grid structure is in developing stage, existing distribution network can be kept active to minimize the power outage for each load. In case of data center, the system could be active and running while the dc distribution network is being modified. For instance, in the case of a data center, in the meantime, loads can be transferred to the new distribution grid independently. Hybrid micro grid Hybrid micro grid consists of ac and dc grids. Fig 1 shows the structure of proposed hybrid micro grid. In the existing network a secondary dc distribution network is super imposed to increase the reliability, power flow, power quality and efficiency. The dc load can fed from dc network and ac loads can fed by ac network. Based on the geographical requirements the system can either operate in stand alone or grid tied mode at several nodes i.e PCCs. Nano grids can act as a centralized grid. Compared to the size of electrical region considered the size of nano grid is small. The nano grid can 


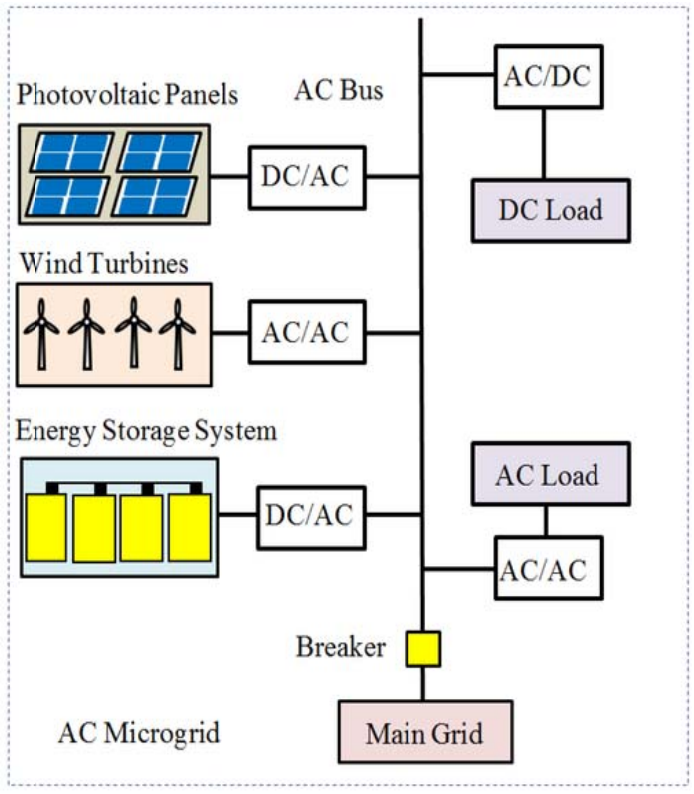

(a)

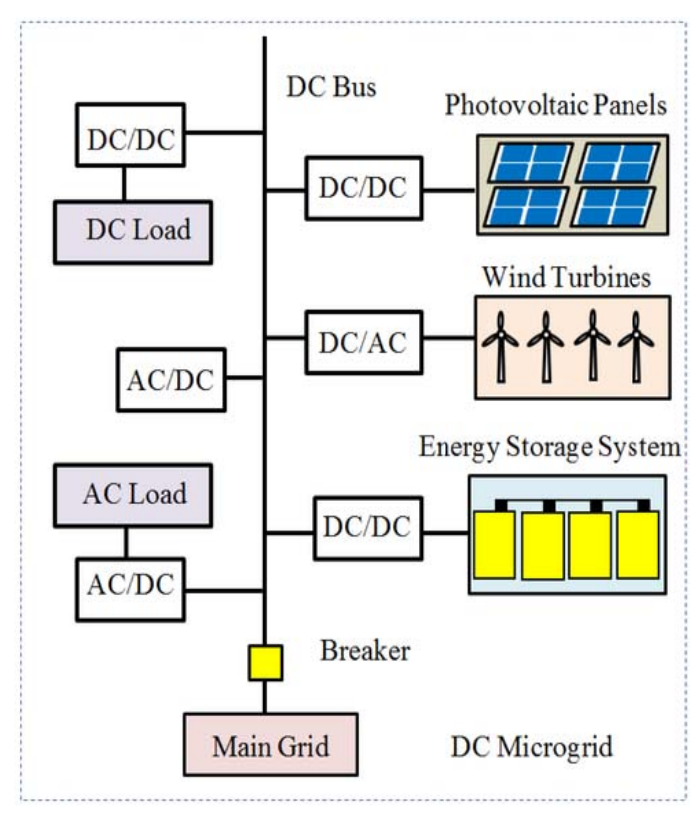

(b)

Fig.1 Structure of proposed hybrid micro grid

access to local distribution generation and storage system and also includes local loads. In the proposed system, each nano-grid is a multi-port power electronic interface (MPEI). MPEI is able to control the power flow between multiple sources and loads and also able to communicate with micro grid controllers. In hybrid micro grid, MPEI is more flexible for energy and power management. For dynamically reconfiguring the distribution network MPEIs acts as a nodes. Due to this, the proposed system can be termed as smart grid. B. Multi Port Power Electronic Interfaces MPEI is a cluster of converters working in harmony with each other. This system consists of DGs and delivers power for local loads and to the de distribution system. This is shown in the Fig. 2.

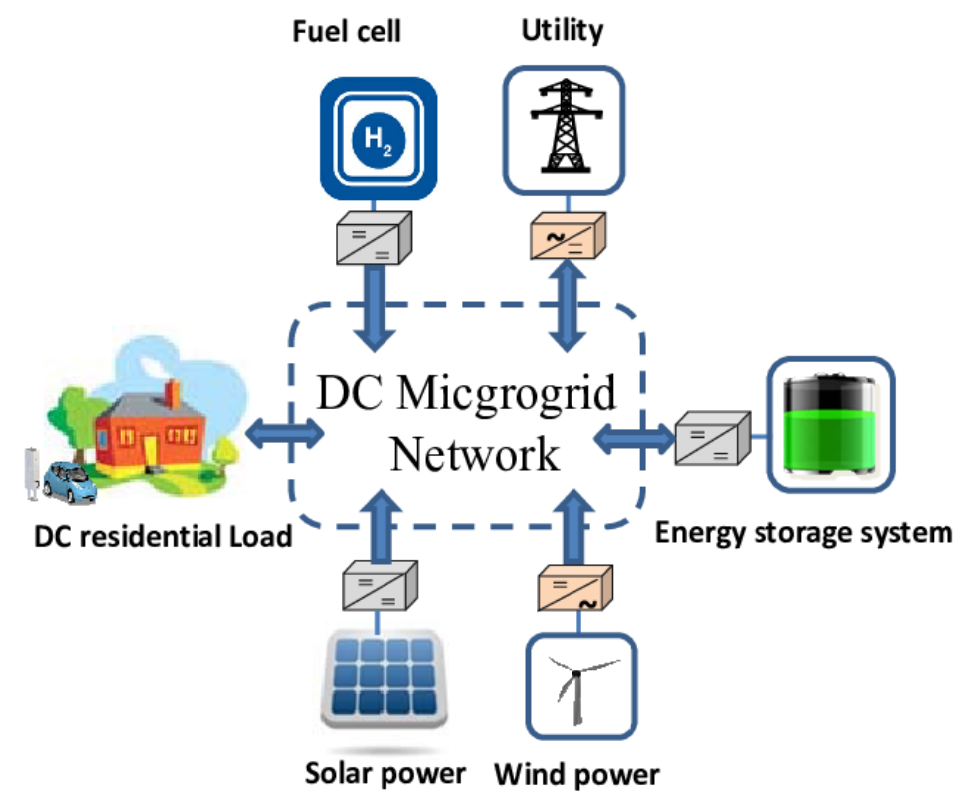

Fig 2 structure of MPEI

B. Model of dc Interface of each MPEI

The use MPEI is introduced in hybrid microgrid. Hence to model a de distribution system of each MPEI id derived here. MPEI model and distribution network are combined for the purpose of studying the hybrid microgrid stability. The internal dc bus voltage is considered to be constant to simplify the modeling purpose. Fig 5 shows the control block diagram of the interface for the dc distribution network. The MPEI model consists of two state equations. First represents the current controller loop. Using control equation, duty cycle generated by this loop can be calculated. 


\section{Micro grid Controller}

Several controllers are used for each individual converter in MPEI based on the requirement. The communication less power sharing techniques can be used to maintain the stability of distribution network. Droop control methods are also used in MPEI to control the distribution network . A micro grid coordinator is used to assure the stability of dc bus and to control the micro grid. The controller monitors the condition of micro grid and the power flow in ac and dc buses. Using dynamic dispatch of reference power commands the stability of ac and dc buses can be maintained by the controller.

\section{MODELLING OF DC MICROGRID}

The model of micro grid consists of model of nodes and distribution network. The MPEI model is derived in. 20 $\mathrm{kHz}$ base frequency with averaging method is derived in this model. For single switching frequency the generalized averaging method is developed. This can be also able to study the behavior of the system at harmonics. Each dc unit have an identical switching frequency. Hence more general form of averaging technique is needed for stability analysis.It is assumed that the frequency of variations of duty cycle is less than the switching frequency for simplifying the Fourier transform calculation. The same assumption is made for generalized averaging method.

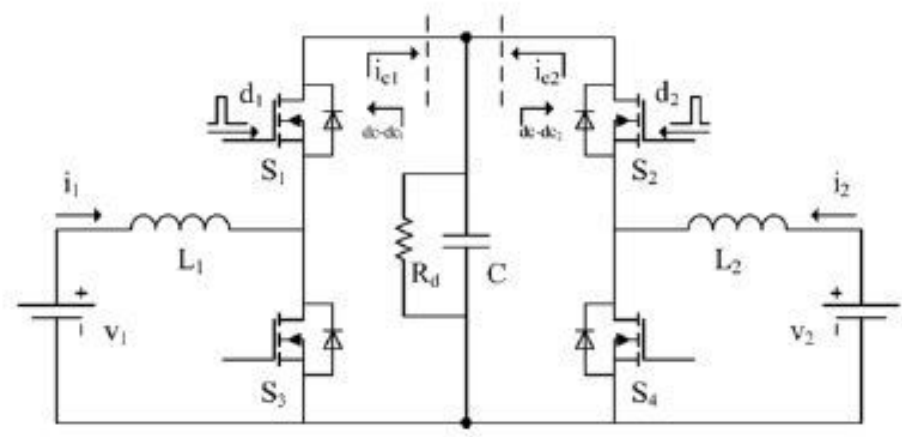

Fig. 3 Back to Back dc-dc switching converter

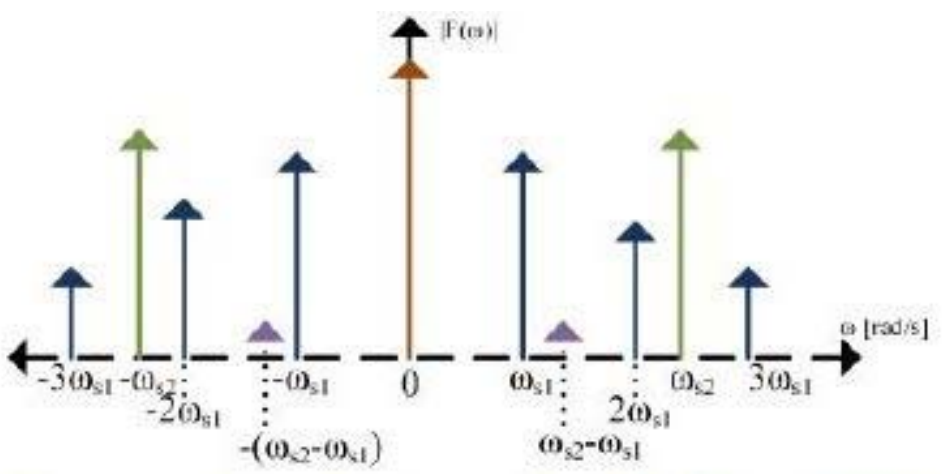

Fig. 4 harmonics contents of the converter in fig 3

In the power electronic converters while considering the non line arities, harmonics in the state variables is not limited to the switching frequency. Each product of these functions result in frequency modulation of all existing harmonics and so results in new harmonics. These offspring harmonics are in the form of linear combinations of the previous harmonics. Thus, based on the switching frequencies of the system, the state variables will have all of the linear combinations of these switching frequencies. The energy of these harmonics is negligible in power electronic converters. To clear this concept, back to back dc to dc converters are used as shown in the fig. 3. In an average dc-dc converter, it is designed to have a steady dc output voltage. Thus, dc is the principal harmonic. Fig. 4 shows an plot of harmonics contents in the back-to-back converter of Fig. 3. In practice, filtering equipments are used to reduce the harmonics though some harmonic remain dominant. Thus, a set of dominant harmonics can be defined for modeling the system with a satisfactory accuracy. 


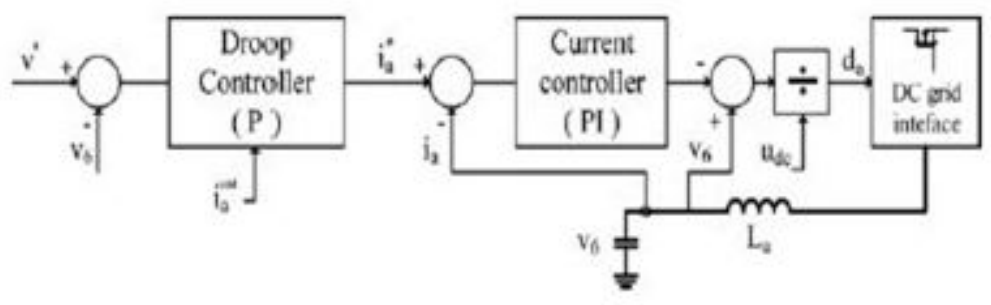

Fig 5 control block diagram of the interface for the dc distributions network

To ensure the stability of parallel connected voltage sources in grid application, droop controller is implemented.

Model of Distribution Network Using $\Pi$ model with lumped parameters, de distribution network is modeled.

Where $\mathrm{L} 7$ is the inductance of a transmission line between the two bus bars. v6 and $\mathrm{v} 7$ are the voltages of the bus bar B6 and B7. C6 is the sum of lumped capacitance of the transmission line and the input capacitance of the MPEI.

\section{STABILITY ANALYSIS USING EXTENDED AVERAGING METHOD}

In order to study the stability of the system, extended averaging method is introduced. Steady State Analysis of Multi Converter System The cascaded system is simulated to study the accurateness of the extended averaging method in assessment of performance of the system. $36 \mathrm{~V}$ source is connected to the first converter dc-dc1. For $24 \mathrm{~V}$ load dc $-\mathrm{dc} 2$ delivers a constant power of $120 \mathrm{~W}$. The dominant harmonics of this system are dc, $\pm 3 \mathrm{kHz}$ and $\pm 5 \mathrm{kHz}$ and the extended averaging model is resultant of the given set of dominant frequencies.

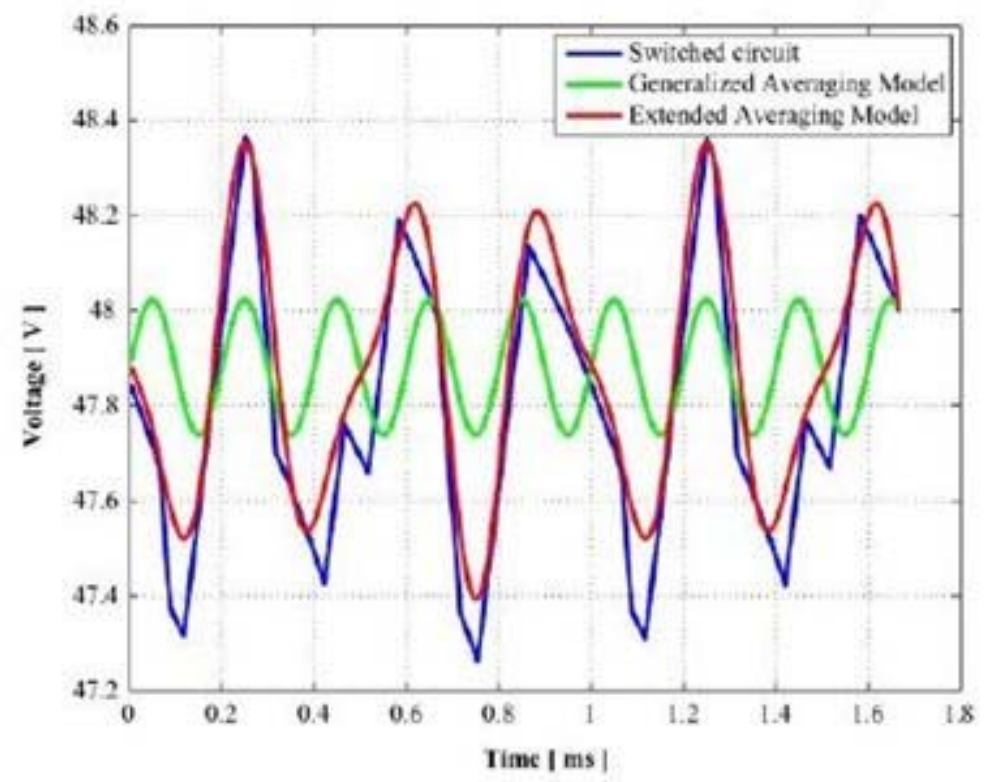

Fig. 6 Signals in time domain

The accuracy of the existing extended averaging method is observed good from the simulation results. Yet, it is not capable to calculate the voltage ripple exactly and it also fails to generate the correct ripple on the dc bus. The proposed extended averaging method is capable to calculate the voltage ripple exactly. Thus for modeling a dc distribution network with multiple switching frequency, this method is implemented. . Stability Assessment Using the Extended Averaging Method From the previous section, closed loop pole zero assessment can be developed. Near the operating point which is the equilibrium point, where the non linear model is linearized. At the equilibrium point the system is stable. It is noted that a system consist of only one operating point. Thus if the operating point is stable the system is stable. From this method, stability assessment of the micro-grid formed between MPEI $a$ and MPEI $b$ can be calculated. 


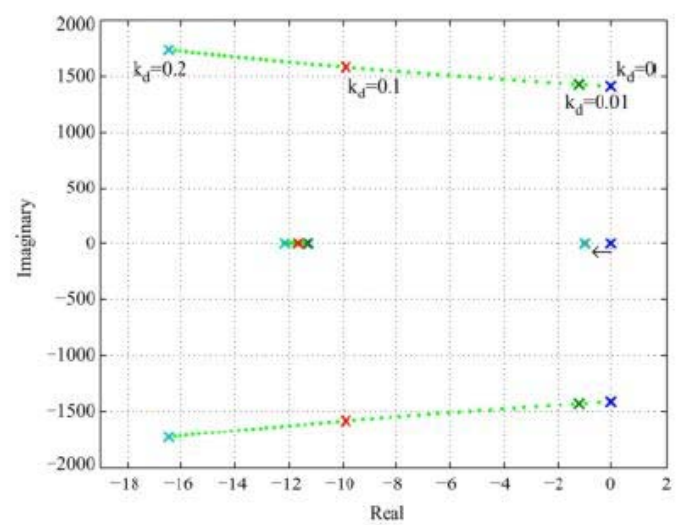

Fig. 7 Pole placement of the characteristic function of the small signal model with respect to variations of the droop coefficient

By using the simplified model, droop coefficient versus pole placement of the characteristic function is shown in the fig. 7. By increasing the droop coefficient, stability of the system is improved. However, in practical applications, the large droop

coefficients are not feasible. This coefficient results in low voltage a regulation which reduces the power quality in a dc distribution network. Dynamic Behavior of Multi Converter System

The dynamic behavior of the system with step load change is studied using the same model. The dc bus voltage is maintained in dc-dc1 converter. Where, dc-dc2 is set to zero. $120 \mathrm{~W}$ is supplied to a fixed load by dc-dc 2 at $0.1 \mathrm{~s}$. The dc bus voltage is shown in the fig. 8 where dc-dc1 operating at $5 \mathrm{kHz}$ and dc-dc 2 operating at $10 \mathrm{kHz}$. Here $200 \mu \mathrm{F}$ dc bus capacitor is used.

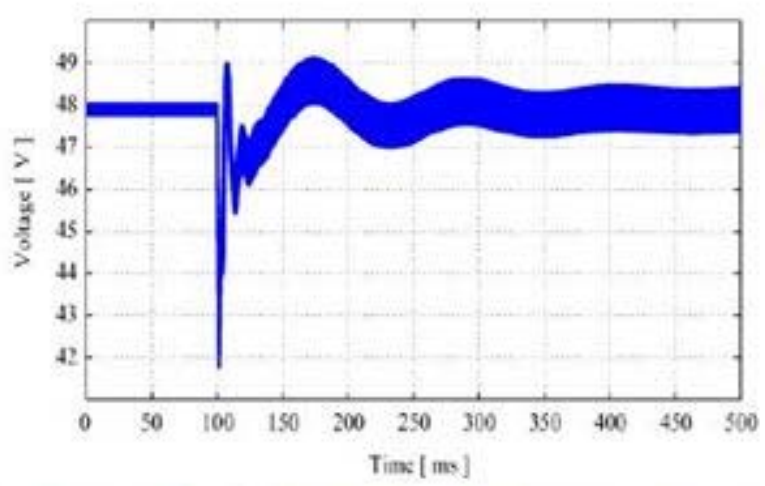

Fig. 8 step response analysis with dc-dc operation at $5 \mathrm{kHz}$ and dc-dc operation at $10 \mathrm{kHz}$

In a data centre, in order to reduce the size of dc-dc converter, it is supplied with digital circuits operating with high frequencies. The switching frequencies of these converters are small i.e. $<20 \mathrm{kHz}$. Fig. 8 shows the simulation result with the switching frequency of the converter increased to $100 \mathrm{kHz}$.

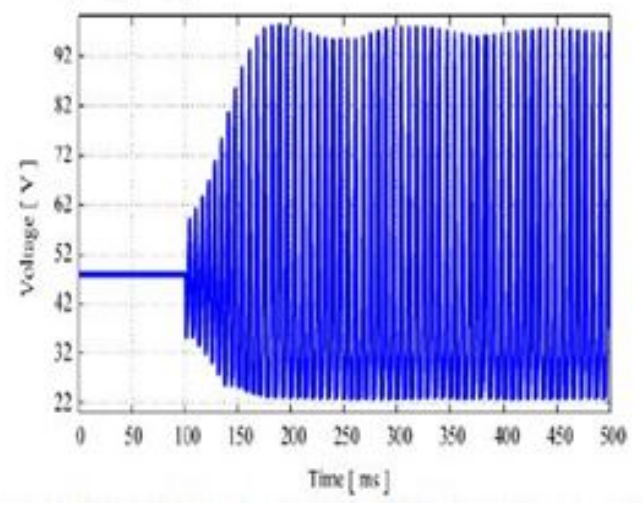

Fig.9 step response analysis with dc-dc operation at $5 \mathrm{kHz}$ and dc-dc operation at $100 \mathrm{kHz}$ 
If the source converters cannot cope with the dynamic response of the load converters, oscillations and limit cycling can occur. This can be observed on the simulated system if the switching frequency of the load converter is increased to $100 \mathrm{kHz}$

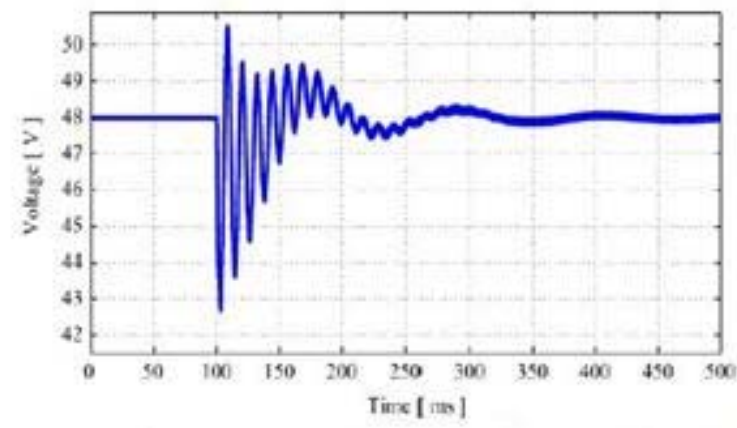

Fig.9 step response analysis with dc-dc operation at $5 \mathrm{kHz}$ and dc-dc operation at $100 \mathrm{kHz}$ with $1000 \mathrm{mf}$ on the DC bus

Hence, when capacitor size is increased, the effect on dc bus voltage is reduced. For this purpose the capacitor of $200 \mu \mathrm{F}$ is increased to $1000 \mu \mathrm{F}$. Fig. 9 shows the step response analysis with dc-dc1 operating at $5 \mathrm{kHz}$ and dc-dc2 operating at $100 \mathrm{kHz}$ with $1000 \mu \mathrm{F}$ on the dc bus. From the simulation result it is identified that the system is stable and $4.7 \mathrm{~A}$ of current is settled at the source converter.

\section{CONCLUSION}

A hybrid microgrid with multi port power electronic interface (MPEI) was implemented in this paper. An extended generalized averaging method is introduced to study the stability of the system. The dc distribution network was also modeled using this method. In the developed model steady state performance model and dynamic pole zero analysis are performed. The accuracy of extended averaging method is illustrated in simulation results. Multi converter system can be modeled using extended averaging method and the results are verified. Using pole zero analysis, the stability assessment can be developed.

\section{ACKNOWLEDGMENT}

I would like to express my special thanks of Mr.Rakesh Oruganti (IJIEMR) as well as Dr. Dharamveer mangal who gave me the golden opportunity to do this wonderful project on the topic (Adaptive High Stability modeling for Analysis of Micro grid for DC Converters), which also helped me in doing a lot of Research and i came to know about so many new things I am really thankful to them. Secondly i would also like to thank my parents and friends who helped me a lot in finalizing this project within the limited time frame.

\section{REFERENCE}

[1] Hua Li, Zhenkui Wu, Jihong Zhang and Hanshan Li, -Wind-Solar-Storage Hybrid Micro grid Control Strategy Based on SVPWM Converterl Journal Of Networks, Vol. 9, No. 6, June 2014

[2] C.-H. Lo and N. Ansari, -Decentralized controls and communications for autonomous distribution networks in smart grid, $\|$ IEEE Trans. Smart Grid, vol. 4, no. 1, pp. 66-77, Mar. 2013.

[3] G. A. Pagani and M. Aiello, - Towards decentralization: A topological investigation of the medium and low voltage grids,ll IEEE Trans. Smart Grid, vol. 2, no. 3, pp. 538-547, Sep. 2011.

[4] Josep M. GuerreroJuan C. Vasquez, José Matas, Luis García de Vicuña, and Miguel Castilla - Hierarchical Control of DroopControlled AC and DC Microgrids-A General Approach Toward Standardizationll IEEE Transactions On Industrial Electronics, Vol. 58, No. 1, January 2011.

[5] Pourya Shamsiand Babak Fahimi, - Dynamic Behavior of Multiport Power Electronic Interface Under Source/Load Disturbances\| IEEE Transactions On Industrial Electronics, Vol. 60, No. 10, October 2013.

[6] H. Behjati and A. Davoudi, -A multi-port dc-dc converter with independent outputs for vehicular applications,ll in Proc.IEEE Veh. Power Propulsion Conf. (VPPC), 2011, pp. 1-5.

[7] Charles K. Sao, and Peter W. Lehn, "Control and Power Management of Converter Fed Microgrids\| IEEE Transactions On Power Systems, Vol. 23, No. 3, August 2008

[8] Yunwei Li, D. Mahinda Vilathgamuwa and Poh Chiang Loh "Design, Analysis, and Real-Time Testing of a Controller for Multibus Microgrid Systemll IEEE Transactions On Power Electronics, Vol. 19, No. 5, September 2004.

[9] Amr Ahmed A. Radwan, and Yasser Abdel-Rady I. Mohamed - Assessment and Mitigation of Interaction Dynamics in Hybrid AC/DC Distribution Generation Systemsll IEEE Transactions On Smart Grid, Vol. 3, No. 3, September 2012.

[10] Sungwoo Bae and Alexis Kwasinsk - Dynamic Modeling and Operation Strategy for a Microgrid With Wind and Photovoltaic Resourcesll IEEE Transactions On Smart Grid, Vol. 3, No. 4, December 2012. 